\title{
Nonlinear Control for Reconfiguration of Unmanned-Aerial-Vehicle Formation
}

\author{
Atilla Dogan* and Sriram Venkataramanan ${ }^{\dagger}$ \\ University of Texas at Arlington, Arlington, Texas 76019
}

\begin{abstract}
The design of a nonlinear controller to reconfigure a formation of a group of unmanned aerial vehicles (UAVs) is described. Reconfiguration of the formation might be needed to maintain the efficiency of the formation. Nonlinear six-degree-of-freedom, rigid-body, equations of motion developed in the virtual leader (VL)'s frame are used to model the UAVs in the formation. The formulation of the formation flight in VL frame enables the formationkeeping and formation reconfiguration to be treated in the same framework. The nonlinear equations of motion contain the wind effect terms and their time derivatives to represent the aerodynamic coupling involved in close formation flight. These wind terms are obtained by using an averaging technique that computes the effective induced wind components and wind gradients in the UAV's body frame. Dynamics of the engine and the actuators are also included in the study. An algorithm that generates a safe and feasible trajectory, given the current position and the position to go to, has been developed. A combination of integral control, optimal LQR design, and nonlinear state feedback linearization is used in the design of the position-tracking controller. Simulation results demonstrate that the controller is capable of producing a smooth reconfiguration without using the information of the vortex-induced wind effects on the follower UAV.
\end{abstract}

\section{Introduction}

$\mathbf{T}$ HE advent of unmanned aerial vehicles (UAVs) into the aviation arena has led to extensive research activities on the design and control of autonomous UAVs to achieve specific mission goals. One of the problems of particular interest to researchers has been the automatic control of a group of UAVs flying in close formation. ${ }^{1,2}$ Most of the research done in the recent past has been focused on the coordination and stationkeeping of multiple UAVs so as to maintain the relative separations and orientations between the UAVs in the formation (see Ref. 3 for relevant publications) and to track desired flight trajectories (see Ref. 4 for related publications). But very little study has been done about the reconfiguration of UAV formation. ${ }^{5,6}$ The reconfiguration might be needed to obtain nearoptimal formation performance in the event of a failure, flight-path restriction, or even the total loss of an aircraft. Specifically, failures such as in one or more communication channels ${ }^{6}$ might necessitate repositioning of aircraft in order to maintain or maximize the overall benefits from the formation flight. Such failures do not affect the control of the individual UAVs in the formation, particularly the ones undergoing reconfiguration. Several theoretical techniques such as graph theory, reconfiguration maps, Dijkstra algorithm, ${ }^{5}$ or functional optimization ${ }^{7}$ have been developed to define the new/optimal positions to be occupied by the UAVs in the formation. However, the six-degree-of-freedom (DOF), nonlinear dynamics involved in the actual repositioning of the UAVs are yet to be investigated, and the control laws for realizing the reconfiguration maneuvers are yet to be developed. Hence our objective in this paper is to develop a control logic to achieve the commands generated by a reconfiguration algorithm and to test the reconfiguration of a UAV flying in a typical close formation, including the dynamic vortex-induced wind effects.

Presented as Paper 2003-5725 at the AIAA Guidance, Navigation, and Control Conference, Austin, TX, 11-14 August 2003; received 3 March 2004; revision received 30 August 2004; accepted for publication 8 September 2004. Copyright (C) 2004 by Atilla Dogan. Published by the American Institute of Aeronautics and Astronautics, Inc., with permission. Copies of this paper may be made for personal or internal use, on condition that the copier pay the $\$ 10.00$ per-copy fee to the Copyright Clearance Center, Inc., 222 Rosewood Drive, Danvers, MA 01923; include the code 0731-5090/05 \$10.00 in correspondence with the CCC.

*Assistant Professor, Department of Mechanical and Aerospace Engineering. Member AIAA.

Graduate Student, Department of Mechanical and Aerospace Engineering. Student Member AIAA.
The remainder of this paper is organized as follows. Section II discusses the modeling considerations involved in our study. It includes both the overall formation modeling and the nonlinear six-DOF modeling of individual UAVs in the formation. Section II.C explains how the wind and wind gradient terms in the equations of motion are computed from the nonuniform vortex field. Section III lists the considerations involved in the generation of a smooth and feasible trajectory for reconfiguration. The control design approach has been illustrated in Sec. IV. Finally, the results of the simulation study have been discussed in Sec. V. In this paper, all of the vectors are expressed according to the vectrix formalism as adopted in Ref. 8.

\section{Modeling}

We consider the formation to be composed of a leader UAV followed by a group of follower UAVs. Basically we have three types of modeling involved in our study: 1) the overall formation modeling, 2) the modeling of the individual UAVs, and 3) vortex effect modeling (i.e., the aerodynamic interaction between the leader and the follower UAV).

In the development of the dynamic model for UAV formation in this paper, there are mainly four basic reference frames involved: 1) inertial frame, 2) virtual-leader (VL) frame, 3) body frame of each UAV, and 4) wind frame of each UAV. The inertial frame is on the ground, and its $z$ axis is pointing downward. The VL frame is always aligned with the inertial frame and moves along the desired trajectory of the formation. In other words, the axes of the VL frame are always parallel to those of the inertial frame even if the VL frame has a translational motion relative to the inertial frame (Fig. 1). The body frame and the wind frame of each UAV are attached to and move with the c.m. of the UAV. The orientation of the body frame relative to the inertial frame is expressed in the standard 3-21 Euler angles notation. For such cases involving multiple reference frames, vectrix formalism ${ }^{8}$ facilitates an efficient transformation of vectors from one frame to another using the rotation matrices. This feature of the vectrix formalism enables the vector equations to be converted easily into the corresponding matrix equations, which are more suitable for implementation in a simulation software. The vectrix of a frame is defined to be the array of unit vectors of the frame and is denoted by

$$
\left[(\hat{)}]=\left[\begin{array}{l}
\hat{\boldsymbol{a}}_{()} \\
\hat{\boldsymbol{J}}_{()} \\
\hat{\boldsymbol{k}}_{()}
\end{array}\right]\right.
$$




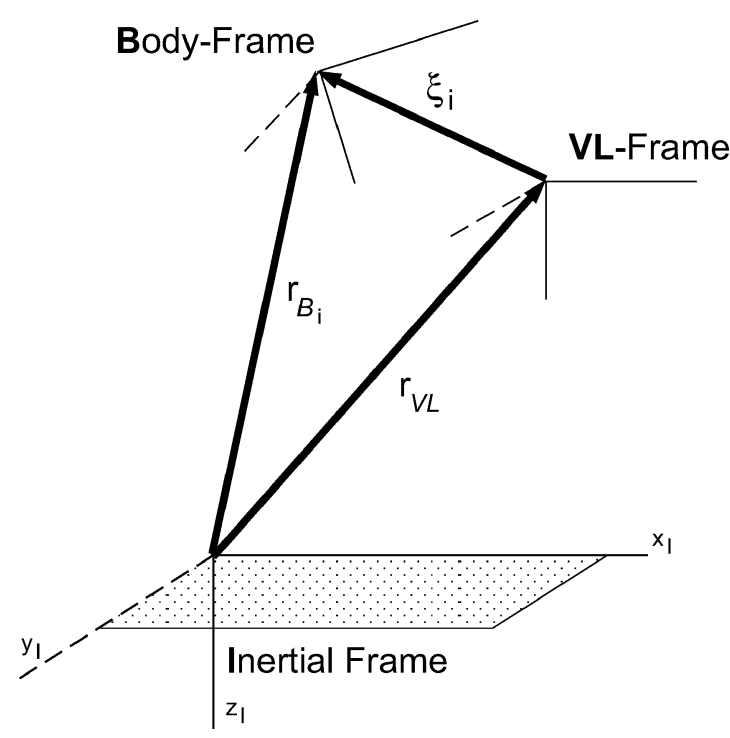

Fig. 1 VL frame used as an intermediate reference frame.

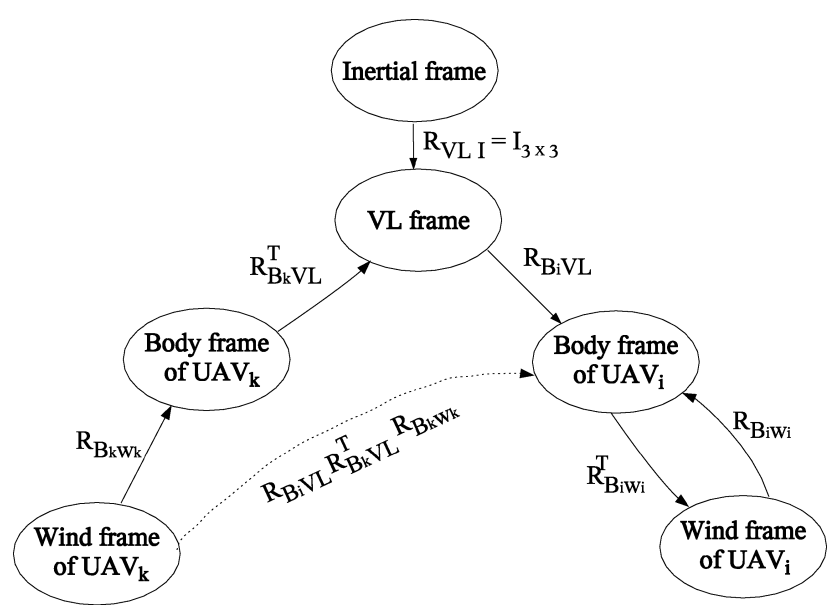

Fig. 2 Various frames and rotation matrices.

Hence, a vector can be written as the product of the transpose of the vectrix of a frame and the representation of the vector in that frame. In this paper, we follow the notation where $A$ is considered to be the representation of a vector $\boldsymbol{A}$ written in the frame in which it is originally defined. All matrices are written in bold font. Figure 2 shows the various reference frames for a two-UAV $\left(\mathrm{UAV}_{i}\right.$ and $\left.\mathrm{UAV}_{k}\right)$ formation and the rotation matrices used to transform a vector from one frame to another.

\section{A. Formation Modeling}

Because one of the applications of this work is the reconfiguration of the UAV formation, a frame fixed to one of the UAVs (either the actual leader or a follower) as the reference frame for the individual UAVs of the formation can lead to a change in the relative positions of all of the UAVs if there comes a need to reposition the UAV whose body/wind frame is chosen as the reference. Hence to avoid such a situation we write the equations of the individual UAVs in the frame of a virtual leader, which is always aligned with the inertial frame and moves along the desired trajectory of the formation. The leader of the formation is supposed to follow the origin of the VL frame. (A similar virtual-leader concept has been outlined in Refs. 5 and 6.) We then select two sets of numbering in our formation model: one to represent the positions relative to the $\mathrm{VL}\left(\operatorname{pos}_{i}\right.$, such as an example shown in Fig. 3 and Table 1) and the other to represent the actual UAVs of the formation $\left(\mathrm{UAV}_{i}\right)$. During normal operation, $\mathrm{UAV}_{i}$ should follow pos ${ }_{j}$, where the values of $i$ and $j$ depend on the flight condition of the formation. Initially, $i=j$. After reconfiguration, $i \neq j$ for some $i$, indicating that those UAVs have been
Table 1 Coordinates of the positions relative to $\mathrm{VL}$ ( $b$, UAV wing span)

\begin{tabular}{lc}
\hline \hline Position no. & $x, y, z$ coordinates \\
\hline 1 & $0,0,0$ \\
2 & $-2 b,-0.8 b, 0$ \\
3 & $-2 b, 0.8 b, 0$ \\
4 & $-4 b,-1.6 b, 0$ \\
5 & $-4 b, 0,0$ \\
6 & $-4 b, 1.6 b, 0$ \\
\hline
\end{tabular}



Fig. 3 Positions of various UAVs relative to the $V L$ in the VL frame.
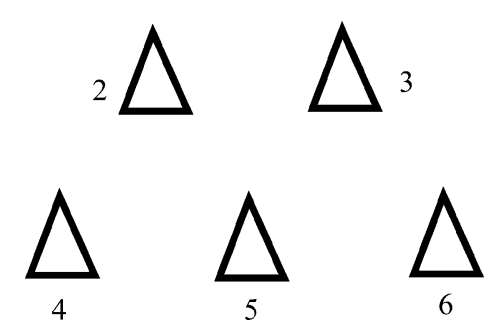

displaced from their original positions in the formation. During the reconfiguration maneuver, $\mathrm{UAV}_{i}$ should follow a trajectory from $\operatorname{pos}_{i}$ to $\operatorname{pos}_{j} ; \operatorname{pos}_{1}$ is the origin of the VL frame, and $\mathrm{UAV}_{1}$ is initially the leader of the formation. Because the origin of the VL frame moves along the desired trajectory, the motion of the origin of the VL frame represents the desired translational motion (position and velocity) of the actual leader. Hence, the actual leader of the formation should follow the $\operatorname{pos}_{1}$, which is made to coincide with the origin of the VL frame (Fig. 3 and Table 1). As the VL moves with time, so do the positions $\operatorname{pos}_{i}$ and hence the actual UAVs. Thus the VL is used to specify the desired trajectory of the overall formation as well as the relative separations of UAVs in the formation. The relative separation between the various positions shown in Fig. 3 and Table 1 should be chosen to obtain the optimal drag benefit from the formation. ${ }^{1}$

\section{B. Modeling of Individual UAVs}

The nonlinear six-DOF equations of motion of the individual UAVs are written in the VL frame.

\section{Translational Motion}

Because we are interested in the positions of the UAVs relative to the VL (the commanded relative position of the actual leader of the formation being the origin) and not their absolute positions with respect to the ground, the translational kinematics equation is written in terms of the position of the aircraft of interest relative to the VL frame and not relative to the inertial frame (Fig. 1). The derivation of these equations is similar to those in Refs. 9 and 10.

We first define the position vectors of the $\mathrm{CM}$ of $\mathrm{UAV}_{i}$ and the origin of the $\mathrm{VL}$ frame relative to the inertial frame as $\boldsymbol{r}_{B_{i}}$ and $\boldsymbol{r}_{\mathrm{VL}}$, respectively. Then, the position vector of the $\mathrm{CM}$ of $\mathrm{UAV}_{i}$ relative to the VL frame is defined as $\boldsymbol{\xi}_{i}$.

Figure 1 implies that

$$
\dot{\boldsymbol{\xi}}_{i}=\dot{\boldsymbol{r}}_{B_{i}}-\dot{\boldsymbol{r}}_{\mathrm{VL}}
$$

We can also write that

$$
\dot{\boldsymbol{r}}_{B_{i}}=\boldsymbol{V}_{\text {inertial }}^{\mathrm{UAV}_{i}}=\boldsymbol{V}_{\frac{\mathrm{UAV}_{i}}{\text { air }}}+\boldsymbol{V}_{\frac{\text { air }}{\text { inertial }}}
$$

where $V_{\mathrm{UAV}_{i} / \text { air }}$ represents the velocity of the CM of the $i$ th UAV relative to the air surrounding it and $\boldsymbol{V}_{\text {air/inertial }}$ denotes the velocity of the local air relative to the inertial frame. The motion of the local air might be caused by the trailing vortices produced by the UAVs (like $\mathrm{UAV}_{k}$ ) leading $\mathrm{UAV}_{i}$, as explained in Sec. II.C, or caused 
by external wind disturbances. Equations (2) and (3) together give the equations of translational kinematics of $\mathrm{UAV}_{i}$ written in matrix form

$$
\dot{\boldsymbol{\xi}}_{i}=\boldsymbol{V}_{\frac{\mathrm{UAV}_{i}}{\text { air }}}+\boldsymbol{V}_{\frac{\text { air }}{\text { inertial }}}-\dot{\boldsymbol{r}}_{\mathrm{VL}}
$$

Note that it is most convenient to write the wind velocity vector affecting $\mathrm{UAV}_{i}$ in its body frame. Furthermore, the velocity of $\mathrm{UAV}_{i}$ with respect the surrounding air should be expressed in terms of conventional variables such as airspeed, angle of attack, and sideslip angle. The following steps are taken to incorporate these changes into the equations of translational kinematics. Let

$$
\boldsymbol{V}_{\text {inertial }}=[\hat{\boldsymbol{I}}]^{T} \boldsymbol{R}_{B_{i} I}^{T} W_{i}=[\widehat{\boldsymbol{V L}}]^{T} \boldsymbol{R}_{B_{i} I}^{T} W_{i}
$$

where

$$
W_{i}=\left[\begin{array}{l}
W_{i_{x}} \\
W_{i_{y}} \\
W_{i_{z}}
\end{array}\right]
$$

and $W_{i_{x}}, W_{i_{y}}$, and $W_{i_{z}}$ are the wind velocity components acting in the $x, y$, and $z$ directions of $\mathrm{UAV}_{i}$ 's body frame. In Eq. (5), $[\hat{\boldsymbol{I}}]^{T}$ and $[\widehat{\boldsymbol{V L}}]^{T}$ denote the vectrix of the inertial and the VL frame, respectively.

Note that because the VL frame is always aligned with the inertial frame, the rotation matrix from the VL frame to the inertial frame is the identity matrix of order three. Hence $[\hat{\boldsymbol{I}}]^{T}$ and $[\widehat{\boldsymbol{V L}}]^{T}$ can be used interchangeably as done in Eq. (5). Another reason to use the body-frame wind velocity components in the equations of motion is that the vortex model used in this paper gives the wind velocity vector in the body frame.

The velocity of the $\mathrm{CM}$ of $\mathrm{UAV}_{i}$ relative to its local air written in terms of its wind-frame axes is

$$
\boldsymbol{V}_{\frac{\mathrm{UAV}_{i}}{\text { air }}}=\boldsymbol{V}_{w_{i}}=\left[\hat{\boldsymbol{w}}_{i}\right]^{T}\left[\begin{array}{c}
V_{i} \\
0 \\
0
\end{array}\right]
$$

where $V_{i}$ is the airspeed of $\mathrm{UAV}_{i}$ and $\left[\hat{\boldsymbol{w}}_{i}\right]^{T}$ is the vectrix of $\mathrm{UAV}_{i}$ 's wind frame.

Writing all of the terms of Eq. (4) in the VL frame and recalling that the rotation matrix from the inertial frame to the VL frame is the identity matrix, we get the translational kinematics equation as

$$
\left[\begin{array}{c}
\dot{x}_{B_{i}} \\
\dot{y}_{B_{i}} \\
\dot{z}_{B_{i}}
\end{array}\right]=\boldsymbol{R}_{B_{i} I}^{T} \boldsymbol{R}_{B_{i} w_{i}} V_{w_{i}}+\boldsymbol{R}_{B_{i} I}^{T} W_{i}-\dot{r}_{\mathrm{VL}}
$$

If $\boldsymbol{R}_{B_{i} I}$ is represented in terms of the Euler angles $\left(\psi_{i}, \theta_{i}, \phi_{i}\right)$ of the $i$ th UAV and $\boldsymbol{R}_{B_{i} w_{i}}$, in terms of its angle of attack $\alpha_{i}$ and the sideslip angle $\beta_{i}$ (Ref. 11), the scalar form of the preceding matrix equation can be obtained as given in Appendix A.

The equations of translational dynamics in matrix form are (see Ref. 9 for the derivation of a similar set of equations)

$$
\begin{aligned}
& {\left[\begin{array}{c}
\dot{V}_{i} \\
\dot{\beta}_{i} \\
\dot{\alpha}_{i}
\end{array}\right]=\mathcal{E}_{i}^{-1} \boldsymbol{S}\left(\omega_{B_{i}}\right) \boldsymbol{R}_{B_{i} w_{i}} V_{w_{i}}+\mathcal{E}_{i}^{-1} \boldsymbol{S}\left(\omega_{B_{i}}\right) W_{i}} \\
& \quad+\left(1 / m_{i}\right) \mathcal{E}_{i}^{-1}\left(\boldsymbol{R}_{B_{i} I} M_{i}+\boldsymbol{R}_{B_{i} w_{i}} A_{i}+P_{i}\right)-\mathcal{E}_{i}^{-1} \dot{W}_{i}
\end{aligned}
$$

where

$$
\mathcal{E}_{i}=\left[\begin{array}{ccc}
\cos \beta_{i} \cos \alpha_{i} & -V_{i} \sin \beta_{i} \cos \alpha_{i} & -V_{i} \cos \beta_{i} \sin \alpha_{i} \\
\sin \beta_{i} & V_{i} \cos \beta_{i} & 0 \\
\cos \beta_{i} \sin \alpha_{i} & -V_{i} \sin \beta_{i} \sin \alpha_{i} & V_{i} \cos \beta_{i} \cos \alpha_{i}
\end{array}\right]
$$

$$
\boldsymbol{S}\left(\omega_{B_{i}}\right)=\left[\begin{array}{ccc}
0 & r_{i} & -q_{i} \\
-r_{i} & 0 & p_{i} \\
q_{i} & -p_{i} & 0
\end{array}\right]
$$

and $\boldsymbol{\omega}_{B_{i}}$, the angular velocity vector of $\mathrm{UAV}_{i}$ in body frame, is written as

$$
\boldsymbol{\omega}_{B_{i}}=\left[\hat{\boldsymbol{B}}_{i}\right]^{T}\left[\begin{array}{c}
p_{i} \\
q_{i} \\
r_{i}
\end{array}\right]
$$

with $\left[\hat{\boldsymbol{B}}_{i}\right]^{T}$ denoting the vectrix of $\mathrm{UAV}_{i}$ 's body frame.

In the derivation of Eq. (9), the resultant force acting on $\mathrm{UAV}_{i}$ has been considered to be the sum of the gravity force vector $\boldsymbol{M}_{i}$ (expressed in the inertial frame), the aerodynamic force vector $\boldsymbol{A}_{i}$ (expressed in the wind frame of $\mathrm{UAV}_{i}$ ), and the propulsive force vector $\boldsymbol{P}_{i}$ (expressed in the body frame of $\mathrm{UAV}_{i}$ ). In general, the representations of the force vectors $\boldsymbol{M}_{i}, \boldsymbol{A}_{i}$, and $\boldsymbol{P}_{i}$ are

$$
M_{i}=\left[\begin{array}{c}
0 \\
0 \\
m_{i} g
\end{array}\right], \quad A_{i}=\left[\begin{array}{c}
-D_{i} \\
-S_{i} \\
-L_{i}
\end{array}\right], \quad P_{i}=\left[\begin{array}{c}
T_{i} \cos \delta_{i} \\
0 \\
-T_{i} \sin \delta_{i}
\end{array}\right]
$$

where $T_{i}$, the thrust, has a fixed inclination $\delta_{i}$ relative to the zero-lift axis, but remains in the aircraft plane of symmetry. The preceding equations are based on the following assumptions: 1) the Earth is flat and nonrotating, and 2) the aircraft weight is constant.

The aerodynamic forces and their coefficients (see Appendix B for a representative set of numerical data needed for simulation) are

$$
\begin{aligned}
D_{i} & =\frac{1}{2} \rho V_{i}^{2} \mathcal{S}_{i} C_{D_{i}} \\
S_{i} & =\frac{1}{2} \rho V_{i}^{2} \mathcal{S}_{i} C_{S_{i}} \\
L_{i} & =\frac{1}{2} \rho V_{i}^{2} \mathcal{S}_{i} C_{L_{i}}
\end{aligned}
$$

where

$$
\begin{aligned}
C_{D_{i}} & =C_{D_{0_{i}}}+C_{D_{\alpha_{i}^{2}}} \alpha_{i}^{2} \\
C_{S_{i}} & =C_{S_{0_{i}}}+C_{S_{\beta_{i}}} \beta_{i}+C_{S_{\delta_{r_{i}}}} \delta_{r_{i}} \\
C_{L_{i}} & =C_{L_{0_{i}}}+C_{L_{\alpha_{i}}} \alpha_{i}+C_{L_{\alpha_{i}^{2}}}\left(\alpha_{i}-\alpha_{\mathrm{ref}_{i}}\right)^{2}+C_{L_{q_{i}}}\left(\bar{c}_{i} / 2 V_{i}\right) q_{\mathrm{rel}_{i}} \\
& +C_{L_{\delta_{e_{i}}}} \delta_{e_{i}}
\end{aligned}
$$

and the air density $\rho$ is calculated as a function of the altitude. ${ }^{12}$

\section{Rotational Motion}

The rotational motion of the individual UAVs is analyzed with reference to the inertial frame (note that the VL frame is always aligned with the inertial frame). Also, the symmetry along the body $x z$ plane has been assumed to be present in all of the UAVs. Hence $I_{x y_{i}}$ and $I_{y z_{i}}$ are considered to be zero.

The rotational kinematics equation in the matrix form is

$$
\dot{\boldsymbol{R}}_{B_{i} I}=\boldsymbol{S}\left(\omega_{B_{i}}\right) \boldsymbol{R}_{B_{i} I}
$$

where $\boldsymbol{S}\left(\omega_{B_{i}}\right)$ and $\omega_{B_{i}}$ are as given in Eqs. (11) and (12).

The rotational dynamics equation in the matrix form is

$$
\boldsymbol{I}_{i} \dot{\omega}_{B_{i}}=S\left(\omega_{B_{i}}\right) \boldsymbol{I}_{i} \omega_{B_{i}}+\mathcal{T}_{i}
$$

where $\mathcal{T}_{i}$ is the total external moment vector of $\mathrm{UAV}_{i}$ expressed about its c.m.:

$$
\mathcal{T}_{i}=\left[\hat{\boldsymbol{B}}_{i}\right]^{T}\left[\begin{array}{c}
\mathcal{L}_{i} \\
\mathcal{M}_{i} \\
\mathcal{N}_{i}
\end{array}\right]
$$


In Eq. (21), $\boldsymbol{I}_{i}$ is the inertia matrix about the c.m. of $\mathrm{UAV}_{i}$, given by

$$
\boldsymbol{I}_{i}=\left[\begin{array}{ccc}
I_{x x_{i}} & 0 & I_{x z_{i}} \\
0 & I_{y y_{i}} & 0 \\
I_{x z_{i}} & 0 & I_{z z_{i}}
\end{array}\right]
$$

and $\boldsymbol{\omega}_{B_{i}}$ is the angular velocity vector as written in Eq. (12).

The aerodynamic moments $\mathcal{L}_{i}, \mathcal{M}_{i}$, and $\mathcal{N}_{i}$ appearing in Eq. (22) and their coefficients are

$$
\begin{gathered}
\mathcal{L}_{i}=\frac{1}{2} \rho V_{i}^{2} \mathcal{S}_{i} b_{i} C_{\mathcal{L}_{i}} \\
\mathcal{M}_{i}=\frac{1}{2} \rho V_{i}^{2} \mathcal{S}_{i} \bar{c}_{i} C_{\mathcal{M}_{i}}+T_{i} \Delta z \\
\mathcal{N}_{i}=\frac{1}{2} \rho V_{i}^{2} \mathcal{S}_{i} b_{i} C_{\mathcal{N}_{i}}
\end{gathered}
$$

where

$$
\begin{aligned}
C_{\mathcal{L}_{i}} & =C_{\mathcal{L}_{0_{i}}}+C_{\mathcal{L}_{\delta_{a_{i}}}} \delta_{a_{i}}+C_{\mathcal{L}_{\delta_{r_{i}}}} \delta_{r_{i}}+C_{\mathcal{L}_{\beta_{i}}} \beta_{i} \\
& +C_{\mathcal{L}_{p_{i}}}\left(b_{i} / 2 V_{i}\right) p_{i_{\mathrm{rel}}}+C_{\mathcal{L}_{r_{i}}}\left(b_{i} / 2 V_{i}\right) r_{i_{\mathrm{rel}}} \\
C_{\mathcal{M}_{i}} & =C_{\mathcal{M}_{0_{i}}}+C_{\mathcal{M}_{\alpha_{i}}} \alpha_{i}+C_{\mathcal{M}_{\delta_{e_{i}}}} \delta_{e_{i}}+C_{\mathcal{M}_{q_{i}}}\left(\bar{c}_{i} / 2 V_{i}\right) q_{i_{\mathrm{rel}}} \\
C_{\mathcal{N}_{i}} & =C_{\mathcal{N}_{0_{i}}}+C_{\mathcal{N}_{\delta_{a_{i}}}} \delta_{a_{i}}+C_{\mathcal{N}_{\delta_{r_{i}}}} \delta_{r_{i}}+C_{\mathcal{N}_{\beta_{i}}} \beta_{i} \\
& +C_{\mathcal{N}_{p_{i}}}\left(b_{i} / 2 V_{i}\right) p_{i_{\mathrm{rel}}}+C_{\mathcal{N}_{r_{i}}}\left(b_{i} / 2 V_{i}\right) r_{i_{\mathrm{rel}}}
\end{aligned}
$$

Note that the effect of thrust on pitching moment is taken into account. Also note that the stability derivatives with respect to the rotational velocities are multiplied by $p_{i_{\text {rel }}}, q_{i_{\text {rel }}}$, and $r_{i_{\text {rel }}}$. This is because the linear expansion of the aerodynamic coefficients is based on the values of angular rotation relative to the atmosphere. ${ }^{9}$ Thus, we must use the rotational velocity of $\mathrm{UAV}_{i}$ relative to the atmosphere. Keeping in mind that $p_{i_{\text {rel }}}, q_{i_{\text {rel }}}$, and $r_{i_{\text {rel }}}$ are written in the body frame of $\mathrm{UAV}_{i}$, the equation for calculating the components of the $\mathrm{UAV}_{i}$ rotational velocity relative to the atmosphere, in its body frame, is

$$
\left[\begin{array}{c}
p_{i_{\text {rel }}} \\
q_{i_{\text {rel }}} \\
r_{i_{\text {rel }}}
\end{array}\right]=\left[\begin{array}{c}
p_{i} \\
q_{i} \\
r_{i}
\end{array}\right]-\left[\begin{array}{c}
p_{\text {eff }_{i}} \\
q_{\mathrm{eff}_{i}} \\
r_{\mathrm{eff}_{i}}
\end{array}\right]
$$

where $p_{\text {eff }_{i}}, q_{\mathrm{eff}_{i}}$, and $r_{\text {eff }}$ are assumed to represent the body angular rates induced by the uniform wind gradients acting on $\mathrm{UAV}_{i}$.

\section{Engine Dynamics}

The thrust generated by the engine $T$ is

$$
T=\xi T_{\max }
$$

where $\xi$ denotes the instantaneous throttle setting and $T_{\max }$ is the maximum available thrust, assumed to be a constant in this paper. The engine dynamics is modeled as that of a first order system with time constant $\tau$. Therefore, we have

$$
\dot{\xi}=\left(\xi-\xi_{t}\right) / \tau
$$

where $\xi_{t}$ is the commanded throttle setting $\left(0.1 \leq \xi_{t} \leq 1\right)$.

\section{Actuator Dynamics}

For the present study, only the actuator saturation and rate limit effects are considered. Other dynamics would be included in a future work. The maximum deflection attainable from the aileron, elevator, and rudder actuators is limited to $\pm 20 \mathrm{deg}$. A rate limit of $50 \mathrm{deg} / \mathrm{s}$ is applied to each of the control surface deflections. Likewise, the saturation limits for engine throttle setting are 0.1 (indicating minimum power output from the engine) and 1 (when maximum available thrust is commanded).

\section{Modeling the Vortex and Its Effect}

The wind effect terms constituting the vectors $W_{i}, \dot{W}_{i}$ in Eqs. (9) and (8) and the induced body rates $p_{\mathrm{eff}_{i}}, q_{\mathrm{eff}_{i}}$, and $r_{\text {eff }}$ in Eq. (30) are considered to be based on the uniform wind distribution acting at $\mathrm{UAV}_{i}$ 's c.m., expressed in its body frame. But, the vortexinduced wind field acting on the encountering aircraft is nonuniform in nature. Therefore, to be able to use the standard aircraft equations of motion without doing any modifications there is a need to approximate the nonuniform induced wind components and gradients by equivalent uniform wind and gradients. Once a fairly reasonable approximation can be achieved, the implementation of aerodynamic coupling between aircraft flying in close proximities becomes far more direct and computationally efficient than the conventional procedure, which involves first the calculation of induced forces and moments from the wind distribution, and then inserting these forces and moments in the aircraft dynamics equations. The need for a simple and fairly accurate method of approximating the nonuniform vortex-induced wind field by its uniform equivalent forms the motivation for the material presented in this section.

In our formation-flight model, every UAV (say, $\mathrm{UAV}_{k}$ ) is considered to produce two straight, semi-infinite trailing vortex filaments that induce additional wind velocities on the body of the following UAV (say, $\left.\mathrm{UAV}_{i}\right)$. These vortex-induced wind velocities cause changes in the forces and moments experienced by $\mathrm{UAV}_{i}$. However, instead of attempting to directly estimate the induced forces and moments on the follower, the induced wind velocities [e.g., $\boldsymbol{W}_{i}$ in Eq. (8)] and wind gradients are computed. The induced wind velocities are written as a function of the relative separation as well as the relative orientation between $\mathrm{UAV}_{k}$ and $\mathrm{UAV}_{i}$ using a modified horseshoe vortex model based on the Helmholtz profile. Because the induced wind and wind gradients are nonuniform along the body dimensions of $\mathrm{UAV}_{i}$, an averaging technique is implemented to compute the effective wind and wind gradient as uniform approximations. The effective wind components and gradients are introduced into the nonlinear aircraft equations that include the components of wind and the temporal variation of wind in the body frame [see Eqs. (8), (9), (20), (21), and (30)] to determine the effect on $\mathrm{UAV}_{i}$ 's dynamics. The effect of vortex decay over time is also included in our model. Special care has been taken to accomodate different geometrical dimensions for $\mathrm{UAV}_{k}$ and $\mathrm{UAV}_{i}$ and also to include many useful geometrical parameters of the UAVs like the wing sweep angle, the dihedral angle, and the relative distance between the center of mass of the UAV and the aerodynamic center of the wing, in estimating the vortex effect experienced during formation flight. For further details of the actual vortex model and the averaging technique used to estimate the vortex effect on the follower UAV, see Ref. 13 or Chapter 3 of Ref. 14.

\section{Trajectory Generation}

For both formation-keeping and formation reconfiguration, the UAVs in the formation need commands of reference trajectories and controllers to track the commanded trajectories. The formulation of the formation flight with respect to the VL frame and the derivation of the equations of motion in the VL frame enables the formationkeeping and formation-reconfiguration to be treated in the same framework.

The desired trajectory depends on the phase of the formation flight. In the case of formation stationkeeping, the desired trajectory actually follows the commanded trajectory of the VL frame. This is to ensure that each UAV will maintain its relative position with respect to the other UAVs in the formation as the leader UAV follows the motion of the virtual leader. The motion of the VL frame serves as the commanded inertial position for the actual leader. In other words, the desired trajectory of each aircraft, in the VL frame, is actually just a point that represents its nominal relative separation from the VL.

However, the generation of a reference trajectory for the problem of formation reconfiguration is more complicated than that for stationkeeping. Section III.A discusses the important aspects involved in designing a safe and feasible reconfiguration trajectory. 
In the discussion of this section, $\mathrm{UAV}_{i}$ is assumed to be commanded to move from $\operatorname{pos}_{i}$ to $\operatorname{pos}_{j}$. To achieve a successful reconfiguration, a safe and smooth trajectory from the starting position (i.e., $\operatorname{pos}_{i}$ ) to the final position (i.e., $\operatorname{pos}_{j}$ ) needs to be generated so that we can control the corresponding UAV (i.e., $\mathrm{UAV}_{i}$ ) to move along the commanded trajectory.

There are two important requirements of the reference trajectory generated for formation reconfiguration. The first and foremost requirement is to ensure the safety of the formation. Namely, there should not be any risk of collision between the UAV under reconfiguration and the other UAVs flying in the formation. Furthermore, the wake vortex of the UAV under reconfiguration should not unnecessarily disturb the other stationkeeping UAVs in the formation. Thus, the reference reconfiguration trajectory should give enough vertical clearance to $\mathrm{UAV}_{i}$ from the plane of the formation (the plane in which all of the other UAVs fly) before the actual $x, y$ position change (in the VL frame) is initiated. To satisfy the first requirement, in our simulation study, a vertical clearance of one wing span of $\mathrm{UAV}_{i}$ is commanded before starting the $x, y$ position change. This vertical clearance also saves the reconfiguration trajectory of $\mathrm{UAV}_{i}$ from passing through the vortex-intensive regions near the other UAVs in the formation.

Second, the generated trajectory should be feasible, namely, realizable within the dynamic constraints of $\mathrm{UAV}_{i}$ and the valid region of the model used. Once the vertical safety clearance is secured, a reference trajectory on the plane parallel to the formation plane is generated to take the UAV from the current position (with the same $x, y$ of $\operatorname{pos}_{i}$ ) to the position with the same $x, y$ coordinates of $\operatorname{pos}_{j}$. To satisfy the second requirement, a continuously differentiable smooth trajectory is used. Particularly, the trajectory consists of arcs of two circles connected at the point where they have the same slope, and at the initial and the final position the slopes are parallel to the heading angle of the formation so that there is no discontinuity in the trajectory as seen in Fig. 4. Heading rates on and the radii of the circles are determined from coordinated-turning trimmed flight condition.

Finally, once the $x, y$ position of $\operatorname{pos}_{j}$ is reached, we command the trajectory to slowly reach the plane of the formation, keeping the $x, y$ position unchanged. In the trajectory generator implemented in our simulation model, the speed on the vertical paths and S-path can be specified separately. To further ensure the feasibility of the reference trajectory, especially the transitions between the three phases, the trajectory is smoothened through three fourth-order filters of the form

$$
\left(s^{4}+k_{13} s^{3}+k_{12} s^{2}+k_{11} s+k_{10}\right) y_{c}=k_{10} Y_{c}
$$

where $Y_{c}$ are reference inputs from the trajectory generator, $k_{10}, \ldots k_{13}$ are suitable positive constant parameters, and $s$ denotes the Laplace variable. The order of the filters and the values of the coefficients are chosen so that the control inputs will not be saturated in their rates trying to follow the commanded trajectory. Particularly, the generated $z$ trajectory for $\mathrm{UAV}_{i}$ (which is required first to create the vertical clearance from the formation plane and then to remove it after the S-maneuver) is a combination of step commands. The control actuation rates are related to the initial slope of the commanded signal. With regard to a step input, Fig. 5 shows



Fig. 4 S-maneuver from $x, y$ of $\operatorname{pos}_{i}$ to that of $\operatorname{pos}_{j}$.

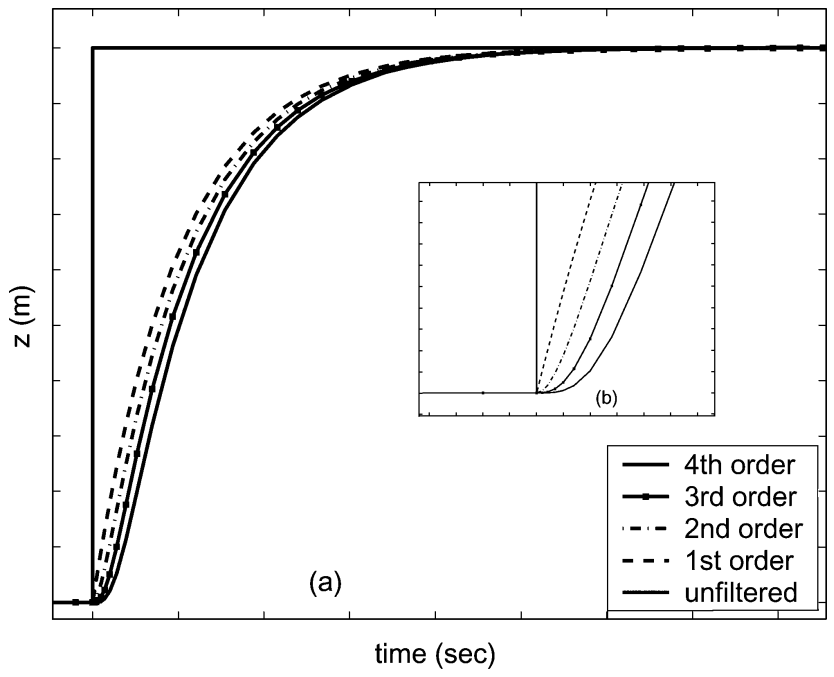

Fig. 5 Effect of filters on step response.

the effect of passing the input signal through linear filters of various orders. Figure 5a shows that the difference in the order of the filters does not cause any significant difference in the speed of the step response, whereas Fig. 5b shows that as the order of the filter is increased the initial slope gets reduced. Hence, for our purpose of reducing the initial slope so as to prevent the rate-limit constraints of the control surface actuators from being violated, linear filters of fourth order are used in the trajectory generation process.

\section{Control Design}

Once the desired trajectory is generated as described in the preceding section, a position-tracking controller is needed to make the $\mathrm{UAV}_{i}$ follow the reference trajectory. During the stationkeeping phase of formation flight, the reference trajectory of each follower UAV (which is a constant point in the VL frame) is determined to maximize the vortex-induced benefits such as drag reduction and thus fuel savings. In a reconfiguration maneuver, the reference trajectory is generated to ensure the overall safety of the formation and a timely repositioning of the UAV. Therefore, it is very important to have a controller that can fly the UAV close to the reference trajectory.

The primary requirement of the control design is the tracking of the generated trajectory, with zero steady-state error in the $x, y, z$ coordinates in the VL frame, under the disturbance of trailing vortex and the acceleration of the VL frame. Meanwhile, the control inputs generated by the controller should not cause significant saturation on the magnitudes and rates of the actuators. Moreover, during the transient, overshoot or undershoot on trajectory response should be minimized to ensure the safety of the formation and also any loss of formation benefits. At the same time, the response of the closed-loop system should be fast enough so that the reconfiguration maneuver is completed as planned and the most efficient spots in the vortex-field are reattained in a timely fashion. Additionally, during the reconfiguration and formation-keeping maneuvers, the angle of attack and the airspeed should not be close to their corresponding stall values. In this regard, very big pitch angle should not be commanded. Finally, to maintain the safety and the efficiency of the formation the bank angle should be small relative to its nominal value.

A combination of nonlinear feedback linearization (inner loop) and integral control linear-quadratic-regulator (LQR) design (outer loop) is employed in designing the position tracking controller satisfying the preceding requirements. A brief schematic of the control system can be seen in Fig. 6.

The step-by-step procedure used in the control design is presented in the following sections. 


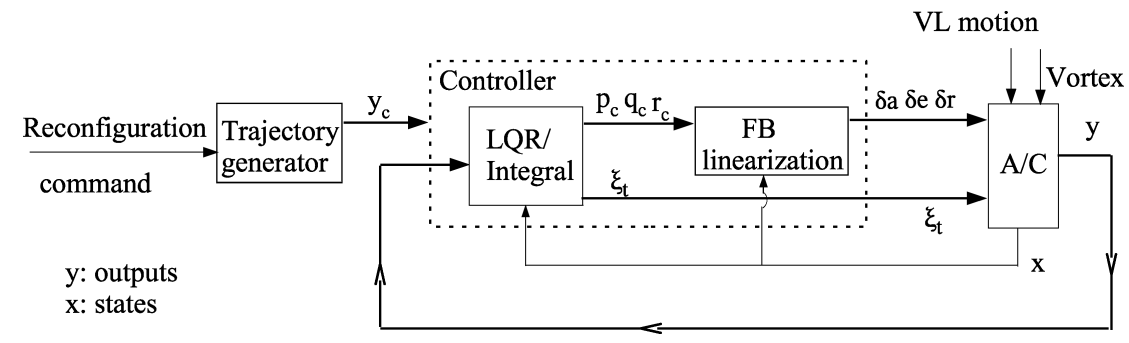

Fig. 6 Schematic diagram showing the inner- and outer-loop controllers.

\section{A. Aircraft Dynamics}

The general aircraft dynamics equations can be put in the form

$$
\begin{gathered}
\dot{\boldsymbol{x}}=f(\boldsymbol{x}, \boldsymbol{W}, \dot{\boldsymbol{W}})+g(\boldsymbol{x}) \boldsymbol{u}+\boldsymbol{B}_{\mathrm{VL}} \dot{\boldsymbol{r}}_{\mathrm{VL}} \\
\boldsymbol{y}=\boldsymbol{C} \boldsymbol{x}
\end{gathered}
$$

where $\boldsymbol{x}$ is the vector of aircraft states; $f(\boldsymbol{x}, \boldsymbol{W}, \dot{\boldsymbol{W}})$ is a nonlinear function of the aircraft states, parameters, the induced wind vector $\boldsymbol{W}$ and its time derivative $\dot{\boldsymbol{W}} ; g(\boldsymbol{x})$ is a nonlinear function that depends on the aircraft states, parameters; and $\boldsymbol{u}$ is the vector of control inputs. The inertial motion of the VL frame is incorporated in the aircraft's translational kinematics through the last term $\boldsymbol{B}_{\mathrm{VL}} \dot{\boldsymbol{r}}_{\mathrm{VL}}$ in the nonlinear aircraft state equation (34), where $\dot{\boldsymbol{r}}_{\mathrm{VL}}$ is the vector of the velocity components of the $\mathrm{VL}$ frame relative to the inertial frame and

$$
\boldsymbol{B}_{\mathrm{VL}}=\left[\begin{array}{lll}
\mathbf{0}_{3 \times 3} & -\boldsymbol{I}_{3 \times 3} & \mathbf{0}_{7 \times 3}
\end{array}\right]^{T}
$$

where $\boldsymbol{I}_{3 \times 3}$ is the identity matrix of order 3 and $\mathbf{0}_{k \times e}$ is the $k \times e$ zero matrix. In the output equation (35), $\boldsymbol{y}$ is the vector of outputs (states whose tracking errors are to be controlled), and $\boldsymbol{C}$ is the output matrix.

In practice, the wind distribution, such as the velocity components and the wind gradients, on an aircraft is very difficult to measure or estimate. Thus, it would be unrealistic to assume that the wind information is available online for the controller. To accomodate this practical feature, in our control design procedure the controller does not use the information about the vortex-induced wind effect that the aircraft is actually exposed to, during its flight. Thus, the wind terms in the equations of motion are ignored in the control law design. Hence, the dynamics equations become

$$
\begin{gathered}
\dot{\boldsymbol{x}}=f(\boldsymbol{x})+g(\boldsymbol{x}) \boldsymbol{u}+\boldsymbol{B}_{\mathrm{VL}} \dot{\boldsymbol{r}}_{\mathrm{VL}} \\
\boldsymbol{y}=\boldsymbol{C} \boldsymbol{x}
\end{gathered}
$$

The aircraft state vector containing 13 states corresponding to the nonlinear aircraft equations of motion listed in Sec. II.B is written as

$$
\boldsymbol{x}=\left[\begin{array}{lllllllllllll}
V & \beta & \alpha & x & y & z & p & q & r & \psi & \theta & \phi & \xi
\end{array}\right]^{T}
$$

Because the purpose of the controller is to make the aircraft track a reference trajectory specified in the VL frame, the outputs are chosen as $x, y, z$, the components of the position vector of the aircraft written in the VL frame. Hence

$$
\boldsymbol{y}=\left[\begin{array}{lll}
x & y & z
\end{array}\right]^{T}
$$

The inputs are the aileron, elevator, rudder deflections, and the engine throttle setting. Therefore, the control vector is

$$
\boldsymbol{u}=\left[\begin{array}{llll}
\delta a & \delta e & \delta r & \xi_{t}
\end{array}\right]^{T}
$$

\section{B. Feedback Linearization}

To facilitate the design process and to obtain a relatively simple control law, the timescale decomposition approach is adopted by considering the rotational dynamics as the fast dynamics. Thus, in the equations of rotational dynamics the body angular rates $(p, q, r)$ are treated as outputs. The rotational dynamics equations are written as

$$
\dot{\boldsymbol{p}}=f_{p}\left(\boldsymbol{x}_{r}, \boldsymbol{p}\right)+g_{p}\left(\boldsymbol{x}_{r}, \boldsymbol{p}\right) \boldsymbol{u}_{\delta}
$$

where $\boldsymbol{p}=\left[\begin{array}{lll}p & q & r\end{array}\right]^{T}, \boldsymbol{u}_{\delta}=\left[\begin{array}{lll}\delta a & \delta e & \delta r\end{array}\right]^{T}$, and $\boldsymbol{x}_{r}$ consist of the remainder of the states. Let the pseudoinput be

$$
\boldsymbol{v}=f_{p}\left(\boldsymbol{x}_{r}, \boldsymbol{p}\right)+g_{p}\left(\boldsymbol{x}_{r}, \boldsymbol{p}\right) \boldsymbol{u}_{\delta}
$$

Thus, the rotational dynamics becomes linear in terms of $\boldsymbol{p}$ and $\boldsymbol{v}$ :

$$
\dot{p}=v
$$

Now, for the feedback linearized rotational dynamics a linear controller for pseudocontrol input $\boldsymbol{v}$ is designed to track the commanded angular rates $\boldsymbol{p}_{c}$. To guarantee zero steady-state error in response to commanded step signals in the presence of disturbance, a proportional-integral control is used. For the integral control, the system is augmented with the states that are integrals of the errors of the output:

$$
\dot{\boldsymbol{w}}=\boldsymbol{p}-\boldsymbol{p}_{c}
$$

With the augmentation states, the system becomes

$$
\left[\begin{array}{c}
\dot{\boldsymbol{p}} \\
\dot{\boldsymbol{w}}
\end{array}\right]=\left[\begin{array}{ll}
\mathbf{0}_{3 \times 3} & \mathbf{0}_{3 \times 3} \\
\boldsymbol{I}_{3 \times 3} & \mathbf{0}_{3 \times 3}
\end{array}\right]\left[\begin{array}{c}
\boldsymbol{p} \\
\boldsymbol{w}
\end{array}\right]+\left[\begin{array}{c}
\boldsymbol{I}_{3 \times 3} \\
\mathbf{0}_{3 \times 3}
\end{array}\right] \boldsymbol{v}+\left[\begin{array}{c}
\mathbf{0}_{3 \times 3} \\
-\boldsymbol{I}_{3 \times 3}
\end{array}\right] \boldsymbol{p}_{c}
$$

A proportional feedback law with a feedforward derivative term is used for the augmented system, namely,

$$
\boldsymbol{v}=-\boldsymbol{K}_{p}\left(\left[\begin{array}{l}
\boldsymbol{p} \\
\boldsymbol{w}
\end{array}\right]-\left[\begin{array}{c}
\boldsymbol{p}_{c} \\
\mathbf{0}_{3 \times 1}
\end{array}\right]\right)+\dot{\boldsymbol{p}}_{c}
$$

The proportional feedback gain matrix $\boldsymbol{K}_{p}$, which is of order $3 \times 6$, is computed using LQR with the following cost index:

$$
J_{P}=\int \boldsymbol{P}^{T} \boldsymbol{Q}_{P} \boldsymbol{P}+\boldsymbol{v}^{T} \boldsymbol{R}_{P} \boldsymbol{v}
$$

where $\boldsymbol{P}=\left[\begin{array}{ll}\boldsymbol{p} & \boldsymbol{w}\end{array}\right]^{T}$. The weighting matrices $\boldsymbol{Q}_{P}, \boldsymbol{R}_{P}$ are chosen as $\boldsymbol{I}_{6 \times 6}$ and $\boldsymbol{I}_{3 \times 3}$, respectively, so that the speed of the response is fast enough for the timescale decomposition approach with the assumption that the rotational dynamics is the fast dynamics. Furthermore, the control surface deflections will not hit their corresponding saturation limits.

Equations (43) and (47) together result in the nonlinear feedback linearization control law for the control surface deflections to track the commanded signals for the outputs $\left(p_{c}, q_{c}, r_{c}\right)$ :

$$
\boldsymbol{u}_{\delta}=g_{p}^{-1}\left[-\boldsymbol{K}_{p}\left(\left[\begin{array}{l}
\boldsymbol{p} \\
\boldsymbol{w}
\end{array}\right]-\left[\begin{array}{c}
\boldsymbol{p}_{c} \\
\mathbf{0}_{3 \times 1}
\end{array}\right]\right)+\dot{\boldsymbol{p}_{c}}-f_{p}\right]
$$


Because we have derivative feedforward terms from the commanded angular rates, the commanded signals should not have very sharp variation. This is achieved by the filters employed in the trajectory generation module.

\section{System Order Reduction}

In the remaining equations of motion (i.e., translational dynamics, translational kinematics, and rotational kinematics), $(p, q, r)$ are treated as input variables in addition to $\xi_{t}$, the commanded engine throttle setting. Here the assumption is that the controller in Sec. IV.B performs fast enough and gives zero steady-state error. Thus from the original system of order 13, a reduced-order system (of order 10) is obtained. The reduced-order dynamics is written in the form

$$
\begin{gathered}
\dot{\boldsymbol{x}}_{r}=f_{r}\left(\boldsymbol{x}_{r}\right)+g_{r}\left(\boldsymbol{x}_{r}\right) \boldsymbol{u}_{r}+\boldsymbol{B} \mathbf{1}_{\mathrm{VL}} \dot{\boldsymbol{r}}_{\mathrm{VL}} \\
\boldsymbol{y}=\boldsymbol{C}_{r} \boldsymbol{x}_{r}
\end{gathered}
$$

where the reduced state vector is

$$
\boldsymbol{x}_{r}=\left[\begin{array}{llllllllll}
V & \beta & \alpha & x & y & z & \psi & \theta & \phi & \xi
\end{array}\right]^{T}
$$

and the input vector is $\boldsymbol{u}_{r}=\left[\begin{array}{llll}p_{c} & q_{c} & r_{c} & \xi_{t}\end{array}\right]^{T}$. In the linearization, the augmented states for the integrals of the errors in angular rates are ignored. The matrix $\boldsymbol{B} \mathbf{1}_{\mathrm{VL}}$ is given by $\left[\begin{array}{llll}\mathbf{0}_{3 \times 3} & -\boldsymbol{I}_{3 \times 3} & \mathbf{0}_{4 \times 3}\end{array}\right]^{T}$ to include the effect of VL motion in the translational kinematics equation, and the output matrix $\boldsymbol{C}_{r}$ is given by $\left[\begin{array}{llll}\mathbf{0}_{3 \times 3} & \boldsymbol{I}_{3 \times 3} & \mathbf{0}_{3 \times 4}\end{array}\right]$ so as to choose the $x, y, z$ positions in VL frame as the outputs to be tracked.

\section{Linearization of the Reduced System}

The reduced-order system is then linearized around the motion of the VL. The assumption made here is that the VL flies in a steadystate condition. During the linearization, the $\dot{\boldsymbol{r}}_{\mathrm{VL}}$ terms present in the nonlinear state equations get cancelled by the nominal conditions. The resulting linearized system is of the form

$$
\begin{gathered}
\Delta \dot{\boldsymbol{x}}_{r}=\boldsymbol{A} \Delta \boldsymbol{x}_{r}+\boldsymbol{B}_{1} \Delta \boldsymbol{u}_{r}+\boldsymbol{B}_{2} \Delta \dot{\boldsymbol{u}}_{r} \\
\boldsymbol{y}=\boldsymbol{C}_{r} \Delta \boldsymbol{x}_{r}
\end{gathered}
$$

where $\Delta \boldsymbol{x}_{r}$ denotes the vector of perturbations in the state variables and $\Delta \boldsymbol{u}_{r}$ those in the inputs to the reduced-order system. $\boldsymbol{A}$ is the system matrix (of size $10 \times 10$ ), and $\boldsymbol{B}_{1}$ is the input matrix (of size $10 \times 4$ ). The derivative term $\Delta \dot{\boldsymbol{u}}_{r}$ appears in Eq. (53) as a result of the presence of $\dot{\boldsymbol{p}}_{c}$ in the control law obtained from the feedback linearization. The matrix $\boldsymbol{B}_{2}$ represents the relation between the various states and the vector $\Delta \dot{\boldsymbol{u}}_{r}\left(=\left[\begin{array}{lllll}\Delta \dot{p}_{c} & \Delta \dot{q}_{c} & \Delta \dot{r}_{c} & \Delta \dot{\xi}_{t}\end{array}\right]^{T}\right)$.

\section{State Transformation}

To be able to apply the standard LQR design to the preceding linearized system, the $\Delta \dot{\boldsymbol{u}}_{r}$ term should be eliminated from the state equation. To do so, we perform a state transformation

$$
\Delta \boldsymbol{z}=\Delta \boldsymbol{x}_{r}-\boldsymbol{B}_{2} \Delta \boldsymbol{u}_{r}
$$

that results in the new system equations in the form

$$
\begin{array}{r}
\Delta \dot{\boldsymbol{z}}=\boldsymbol{A} \Delta \boldsymbol{z}+\left(\boldsymbol{A} \boldsymbol{B}_{2}+\boldsymbol{B}_{1}\right) \Delta \boldsymbol{u}_{r} \\
\boldsymbol{y}=\boldsymbol{C}_{r} \Delta \boldsymbol{z}+\boldsymbol{C}_{r} \boldsymbol{B}_{2} \Delta \boldsymbol{u}_{r}
\end{array}
$$

\section{System Augmentation}

The transformed system is then augmented with four integral states. The first three are the integrals of errors in the positions $(x, y, z)$,

$$
\boldsymbol{w}_{1}=\left[\begin{array}{lll}
\int x-x_{c} & \int y-y_{c} \quad \int z-z_{c}
\end{array}\right]^{T}
$$

and the fourth is the integral of the deviation of the bank angle from its nominal value $\left(w_{2}=\int \Delta \phi\right)$. The reason for including $w_{2}$ as an augmented state is to make the steady-state bank angle equal to its nominal value. The equation for $\dot{w}_{2}$ is written in the form

$$
\dot{w}_{2}=\boldsymbol{C}_{2} \Delta \boldsymbol{x}_{r}
$$

where $\boldsymbol{C}_{2}=\left[\begin{array}{lll}\mathbf{0}_{1 \times 8} & 1 & 0\end{array}\right]$. We cannot add another integrator for the yaw angle because that would make the augmented system uncontrollable. The uncontrollability is because the number of outputs to track in the augmented system would be greater than the number of available control inputs, which is four in this system. Therefore, the augmented system is written as

$$
\begin{gathered}
\Delta \dot{\boldsymbol{x}}_{\text {aug }}=\boldsymbol{A}_{\text {aug }} \Delta \boldsymbol{x}_{\text {aug }}+\boldsymbol{B}_{\text {aug }} \Delta \boldsymbol{u}_{r}+\left[\begin{array}{c}
\mathbf{0}_{10 \times 3} \\
-\boldsymbol{I}_{3 \times 3} \\
\mathbf{0}_{1 \times 3}
\end{array}\right] \Delta \boldsymbol{y}_{c} \\
\boldsymbol{y}=\left[\begin{array}{ll}
\boldsymbol{C}_{r} & \mathbf{0}_{3 \times 4} \\
\boldsymbol{C}_{2} & \mathbf{0}_{1 \times 4}
\end{array}\right] \Delta \boldsymbol{x}_{\text {aug }}+\left[\begin{array}{l}
\boldsymbol{C}_{r} \boldsymbol{B}_{2} \\
\boldsymbol{C}_{2} \boldsymbol{B}_{2}
\end{array}\right] \Delta \boldsymbol{u}_{r}
\end{gathered}
$$

where the augmented state perturbation vector $\Delta \boldsymbol{x}_{\text {aug }}$ is given by

$$
\Delta \boldsymbol{x}_{\mathrm{aug}}=\left[\begin{array}{c}
\Delta \boldsymbol{z} \\
\boldsymbol{w}_{1} \\
w_{2}
\end{array}\right]
$$

the augmented system matrix $\boldsymbol{A}_{\text {aug }}$ is given by

$$
\boldsymbol{A}_{\text {aug }}=\left[\begin{array}{cc}
\boldsymbol{A} & \mathbf{0}_{10 \times 4} \\
\boldsymbol{C}_{r} & \mathbf{0}_{3 \times 4} \\
\boldsymbol{C}_{2} & \mathbf{0}_{1 \times 4}
\end{array}\right]
$$

and the input matrix of the augmented system is expanded as

$$
\boldsymbol{B}_{\mathrm{aug}}=\left[\begin{array}{c}
\boldsymbol{A} \boldsymbol{B}_{2}+\boldsymbol{B}_{1} \\
\boldsymbol{C}_{r} \boldsymbol{B}_{2} \\
\boldsymbol{C}_{2} \boldsymbol{B}_{2}
\end{array}\right]
$$

Note that the augmented state equation (59) contains $\Delta \boldsymbol{y}_{c}$, which is the vector of perturbations in the reference positions to be tracked.

\section{E. LQR Design}

After verifying the stabilizability of the augmented system, a cost index for LQR design is defined using $\Delta \boldsymbol{x}_{\text {aug }}$ as

$$
J=\int \Delta \boldsymbol{x}_{\text {aug }}^{T} \boldsymbol{Q} \Delta \boldsymbol{x}_{\text {aug }}+\Delta \boldsymbol{u}_{r}^{T} \boldsymbol{R} \Delta \boldsymbol{u}_{r}
$$

LQR is used to design a stabilizing controller with zero steady-state error in outputs. The most important requirement on the closed-loop system is to have fast response with little overshoot or undershoot to prevent any risk of collision. To avoid loss of control during the reconfiguration maneuver, the control input saturation should be minimized. Thus, the state and the control weighting matrices [ $\boldsymbol{Q}$ and $\boldsymbol{R}$ in Eq. (64), respectively] are chosen considering the criticality of the states and actuator saturation limits. The second row of $\boldsymbol{Q}$ is particularly weighted more (see Appendix B for the actual numbers used for the simulation) to satisfy the requirement to keep the angle of attack low. The resulting control law is of the form

$$
\Delta \boldsymbol{u}_{r}=-\boldsymbol{K}\left[\begin{array}{c}
\Delta \boldsymbol{z} \\
\boldsymbol{w}_{1} \\
w_{2}
\end{array}\right]
$$

where $\boldsymbol{K}$ is the optimal state feedback gain matrix computed by the LQR method. Note from Eq. (55) that $\Delta \boldsymbol{z}$ includes $\Delta \boldsymbol{u}_{r}$. Thus, we need to substitute $\Delta z$ into the preceding equation to explicitly get the feedback law. Therefore,

$$
\Delta \boldsymbol{u}_{r}=-\left(\boldsymbol{I}_{4 \times 4}-\boldsymbol{K}_{0} \boldsymbol{B}_{2}\right)^{-1} \boldsymbol{K}\left[\begin{array}{c}
\Delta \boldsymbol{x}_{r} \\
\boldsymbol{w}_{1} \\
w_{2}
\end{array}\right]
$$


where $\boldsymbol{K}_{0}$ constitutes the first 10 columns of matrix $\boldsymbol{K}$. Matrix $\left(\boldsymbol{I}_{4 \times 4}-\boldsymbol{K}_{0} \boldsymbol{B}_{2}\right)$ should be verified for invertibility.

The elements of $\Delta \boldsymbol{u}_{r}$, namely, $\Delta p_{c}, \Delta q_{c}, \Delta r_{c}$, and $\Delta \xi_{t}$, are then added to their respective nominal values corresponding to the condition around which the reduced-order system was linearized (in Sec. IV.C.1), to obtain the absolute values of $p_{c}, q_{c}$, and $r_{c}$, which form the reference command for the outputs of the feedback linearization controller to track.

\section{F. Overall Controller}

The control laws from nonlinear feedback linearization and LQR are combined to obtain the overall nonlinear controller for $\left[\begin{array}{llll}\delta a & \delta e & \delta r & \xi_{t}\end{array}\right]^{T}$ to track the commanded $(x, y, z)$ in the VL frame.

Finally, the nonlinear controller is implemented in the nonlinear equations of motion with wind terms (as given in Sec. II.B) to study its performance in the actual formation-flight simulations.

\section{Simulation Results}

The multiloop controller described in this paper is tested in two different formation-flight scenarios with a nonlinear simulation environment developed by using MATLAB ${ }^{\circledR} /$ Simulink. Whereas the aim of the first test (Sec. V.A) is to prove good formation-keeping despite leader's maneuvers, the second test (Sec. V.B) is intended to check the performance of the controller in accomplishing a specific formation reconfiguration.

Both the simulation tests consider only two UAVs flying in formation. The nominal flight condition considered for the UAV formation is a straight-and-level flight with a steady forward velocity of $150 \mathrm{~m} / \mathrm{s}$ at an altitude of $3000 \mathrm{~m}$ above mean sea level. UAV flies at $\operatorname{pos}_{1}$ (see Fig. 3 and Table 1) and $\mathrm{UAV}_{2}$ at $\operatorname{pos}_{6}$, which is in $\mathrm{UAV}_{1}$ 's vortex wake. The aircraft parameters chosen for the simulation represent a typical transport-class aircraft (see Appendix B).

\section{A. Formation Stationkeeping}

The objective of this simulation experiment is to test if $\mathrm{UAV}_{1}$ and $\mathrm{UAV}_{2}$ would adjust themselves to follow and maintain their relative separations and positions relative to the VL frame. Because the commanded trajectory of each UAV is constant (representing its corresponding position) in the VL frame, the simulation also shows the stability of the formation under the effect of two types of disturbances: the trailing vortex and the motion of the VL frame. The VL is commanded a complicated maneuver for a short time, in which small additional step inputs are introduced in its $x, y, z$ velocities, and are later withdrawn after $60 \mathrm{~s}$. This maneuver results in shifting the inertial position of the VL frame along the $x, y, z$ directions as shown in Fig. 7. Note that while the VL is moved continuously along the inertial $y$ and $z$ directions, its speed in the $x$ direction is also higher than $150 \mathrm{~m} / \mathrm{s}$. (This is not seen clearly as a slope change in Fig. 7 because of the scaling.) At the end of


Fig. 7 Position of the VL frame relative to the inertial frame.
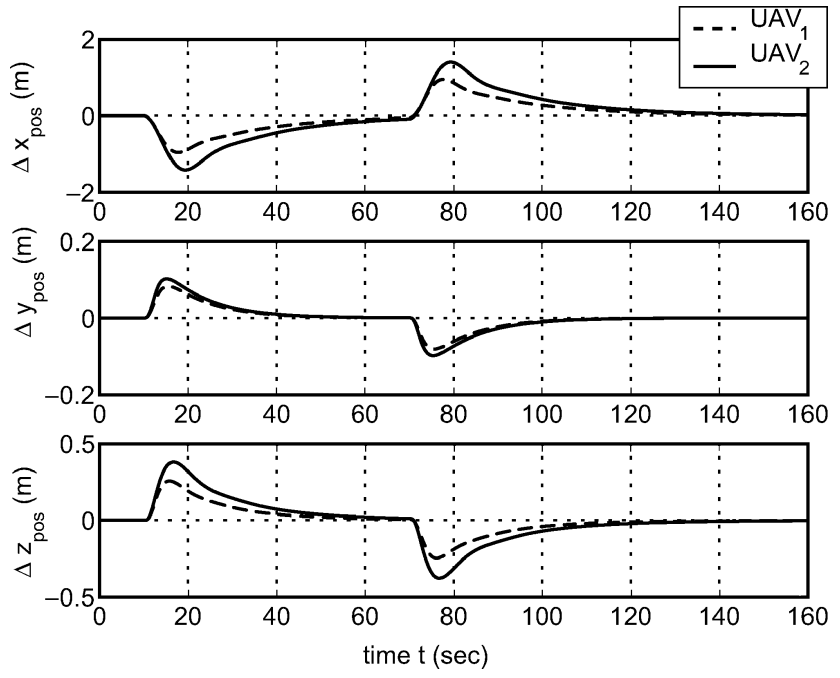

Fig. 8 Deviations of $\mathrm{UAV}_{1}$ and $\mathrm{UAV}_{2}$ from their nominal relative separations.

this maneuver, the VL is moved by about $120 \mathrm{~m}$ toward the left as well as by about $120 \mathrm{~m}$ above while its $x$ velocity is restored to its original value of $150 \mathrm{~m} / \mathrm{s}$. Note that a decrease in $\mathrm{Z}_{\mathrm{VL}}$ represents an increase in altitude.

Figure 8 shows that the UAVs indeed correct their positions in the VL frame to keep pace with the VL's motion. The figure also shows that the deviations of the UAVs from their nominal positions in the VL frame are small and in the same direction. Thus, during the maneuver of the formation any risk of collision is minimal. The follower shows greater deviation from its nominal values and also takes longer to adjust itself, illustrating the difficulty of controlling an aircraft flying in its leader's wake vortex. Moreover, the time response of the control inputs Fig. 9 shows that all of the control surface deflections and the engine throttle setting for both the leader and the follower UAV stay well within their saturation and rate limits. The benefit from formation flight can also be observed from Fig. 9, which clearly demonstrates that the follower needs a lesser throttle setting (and hence engine power) than its leader. Note also that the steady-state level of the throttle setting is lower after the VL maneuver than the level before the maneuver for both leader and follower. This is because by the end of the VL maneuver the altitude of the formation increases by $120 \mathrm{~m}$ where the air density is less.

\section{B. Formation Reconfiguration}

After the stationkeeping performance of the formation is ensured, the simulation of a formation-reconfiguration maneuver is performed. The reconfiguration command is for the $\mathrm{UAV}_{2}$ to move from $\operatorname{pos}_{6}$ to $\operatorname{pos}_{2}$ while the $\mathrm{VL}$ and $\mathrm{UAV}_{1}$ maintain their original steady-state condition. The commanded reference trajectory for reconfiguration (Fig. 10) involves three consecutive phases of motion of $\mathrm{UAV}_{2}$ relative to the VL. In the first phase (Figs. 10 and 11), $\mathrm{UAV}_{2}$ moves below the $x y$ plane of the VL frame by one wing span (which was considered to be sufficient vertical clearance), while still at the $x, y$ coordinates of pos $_{6}$. The second phase involves the S-maneuver in which the $\mathrm{UAV}_{2}$ moves smoothly and continuously on the $X Y$ plane to eventually reach the $x, y$ coordinates of $\operatorname{pos}_{2}$. In the last phase, the $\mathrm{UAV}_{2}$ is commanded to slowly rise back to the altitude of the VL. An additional feature of physical significance included in the simulation is that the $\mathrm{UAV}_{2}$ would wait for a certain time interval between the vertical maneuvers and the $S$-maneuver. The time interval between maneuvers is influenced by the filter time constants used in the trajectory generator. The slower the response time of the filter, the greater the waiting interval between maneuvers should be. The speed of reconfiguration and the interval of waiting between maneuvers can be varied in the simulation. The results are presented for the case when the reconfiguration speed is $15 \mathrm{~m} / \mathrm{s}$, and the interval between maneuvers is $20 \mathrm{~s}$. As mentioned in Sec. III, all of the reference position commands are passed through a fourth-order 

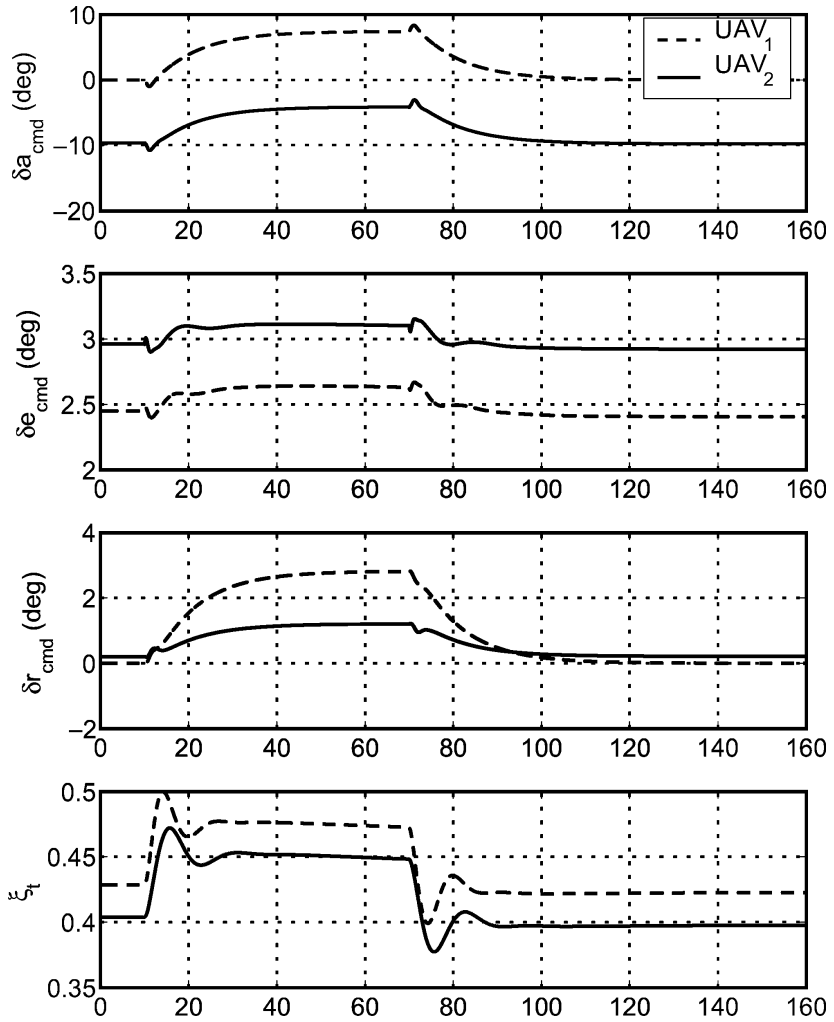

time $t(\mathrm{sec})$

Fig. 9 Control inputs to $\mathrm{UAV}_{1}$ and $\mathrm{UAV}_{2}$ in response to the maneuvering VL.

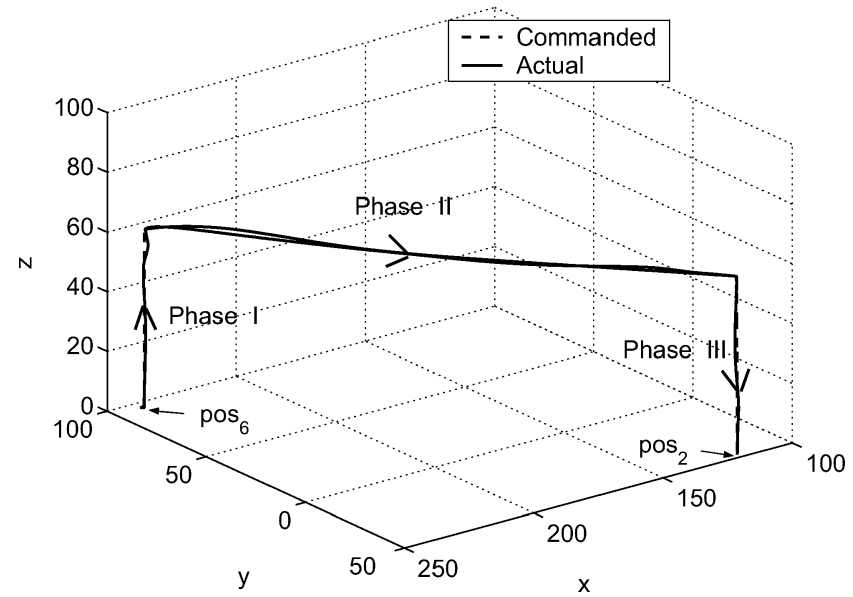

Fig. 10 Reference trajectory for reconfiguration of $U_{A V} V_{2}$ from $\operatorname{pos}_{6}$ to $\operatorname{pos}_{2}$.

filter before being fed to the controller. In Fig. 11, the UAVs were flying in trim condition up to $10 \mathrm{~s}$. The overall reconfiguration takes about $3 \mathrm{~min}$ to be completed. This figure demonstrates that excellent position tracking is achieved throughout the reconfiguration maneuver despite the vortex effects. Also, the UAV is brought to a trim condition at the end of the reconfiguration. At no point during the reconfiguration has there been any risk of collision between $\mathrm{UAV}_{2}$ and $\mathrm{UAV}_{1}$.

Figure 12 shows the effective wind velocity components and gradients experienced by $\mathrm{UAV}_{2}$ during the reconfiguration flight. It is seen that the steady wind (mainly $W_{z}$ ) at the final position is higher than at the initial position of $\mathrm{UAV}_{2}$. This is because $\mathrm{pos}_{2}$ is closer to the vortex-generating actual leader $\left(\mathrm{UAV}_{1}\right)$ than $\operatorname{pos}_{6}$. Also, at pos 2 ,
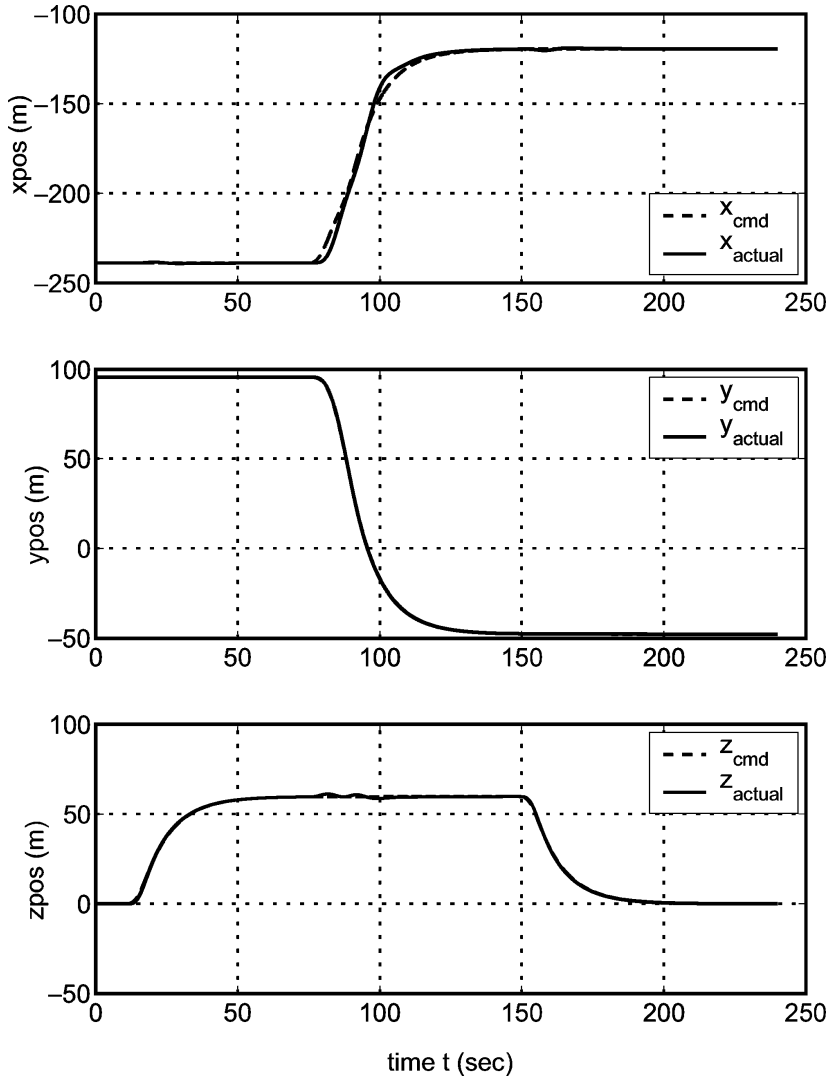

Fig. 11 Time history of commanded and actual positions of $\mathrm{UAV}_{2}$ in VL frame.
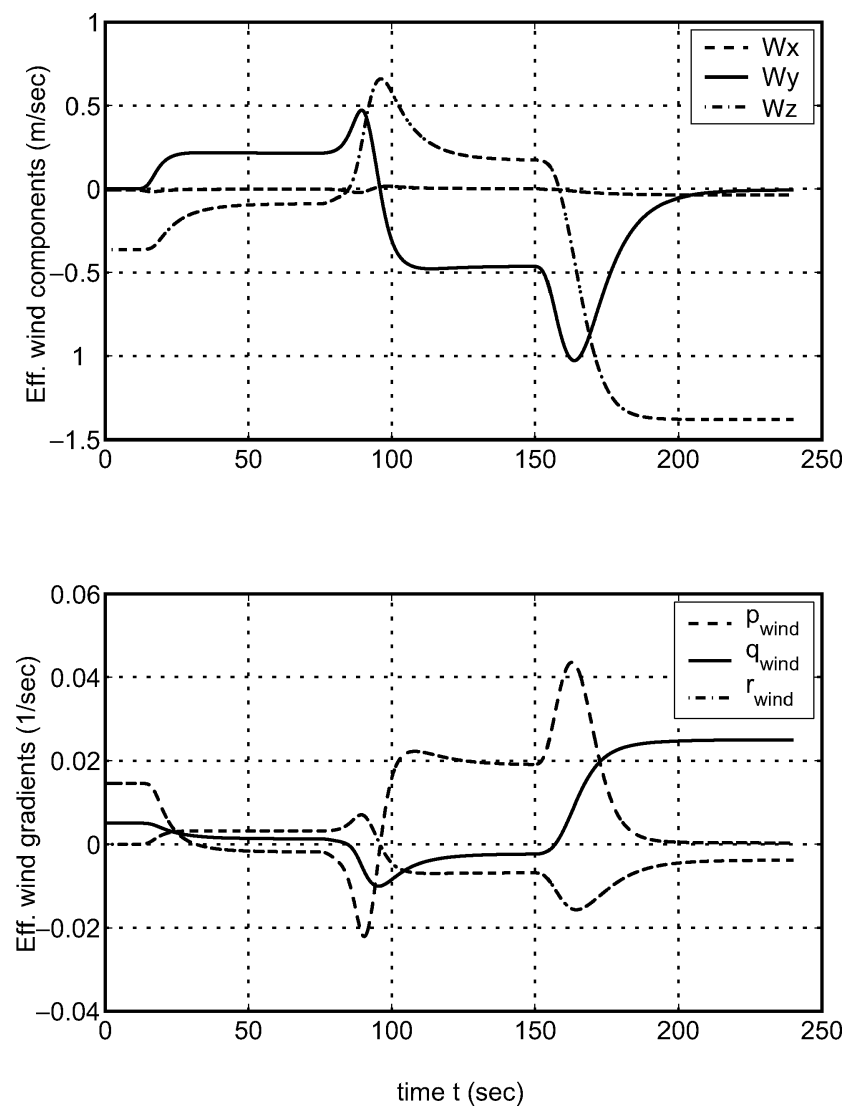

Fig. 12 Effective wind components and gradients induced during reconfiguration. 




Fig. 13 Time history of control surface deflections and the engine throttle setting.

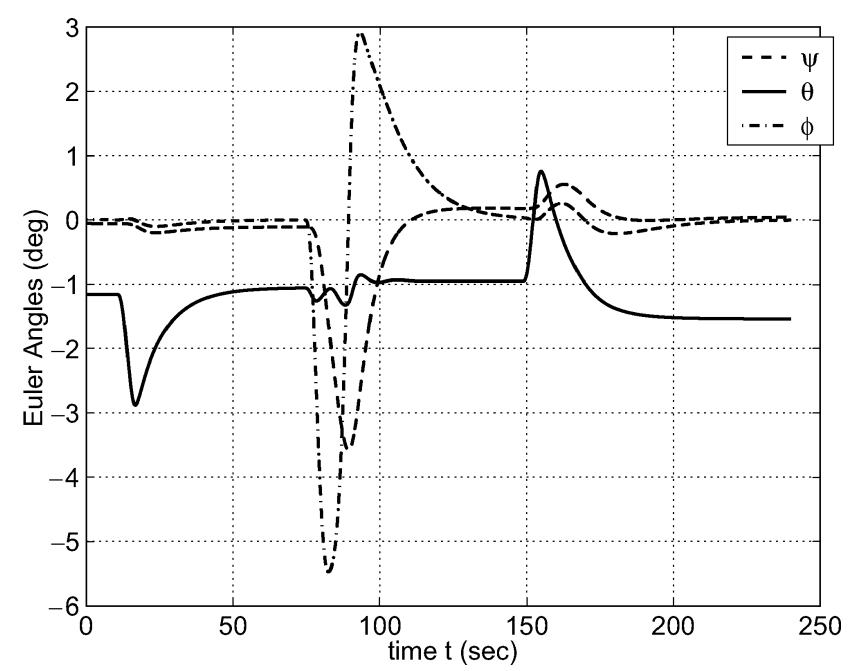

Fig. 14 Variation of the Euler angles of $\mathrm{UAV}_{2}$ during the reconfiguration.

the steady effective wind gradients indicate higher constant rolling and pitching moment induced on $\mathrm{UAV}_{2}$ by the vortices emerging from $\mathrm{UAV}_{1}$.

From Fig. 13, it is seen that the commanded control surface deflections and the throttle setting are smooth and kept well within their saturation limits. It has been verified by observation that the greater the ratio of reconfiguration speed to the velocity of the VL, the greater is the maximum control surface deflection required during the reconfiguration maneuver (especially the transient aileron deflection needed to navigate the S-maneuver). It is also seen that the commanded steady-state aileron and rudder deflections at the end of the reconfiguration are nonzero as a result of the presence of the constant vortex-induced wind gradients that $\mathrm{UAV}_{2}$ is subjected to while flying at $\operatorname{pos}_{2}$.

The Euler angles, the angle of attack, and the sideslip angle encountered by $\mathrm{UAV}_{2}$ during the reconfiguration maneuver are presented in Figs. 14 and 15.

It can be seen from Fig. 14 that the steady-state value of the yaw angle is slightly different from its nominal value. A simulation experiment was carried out to understand the effect of vortex on the steady-state values of the Euler angles. In the case where only the effective wind components were present (the effective wind gradients "turned off"), $\psi$ and $\phi$ both had zero steady-state values. In another case where only the effective wind gradients were present without wind components, $\psi$ had a nonzero steady-state value while $\phi$ had zero steady-state value as in the normal case (both wind com-

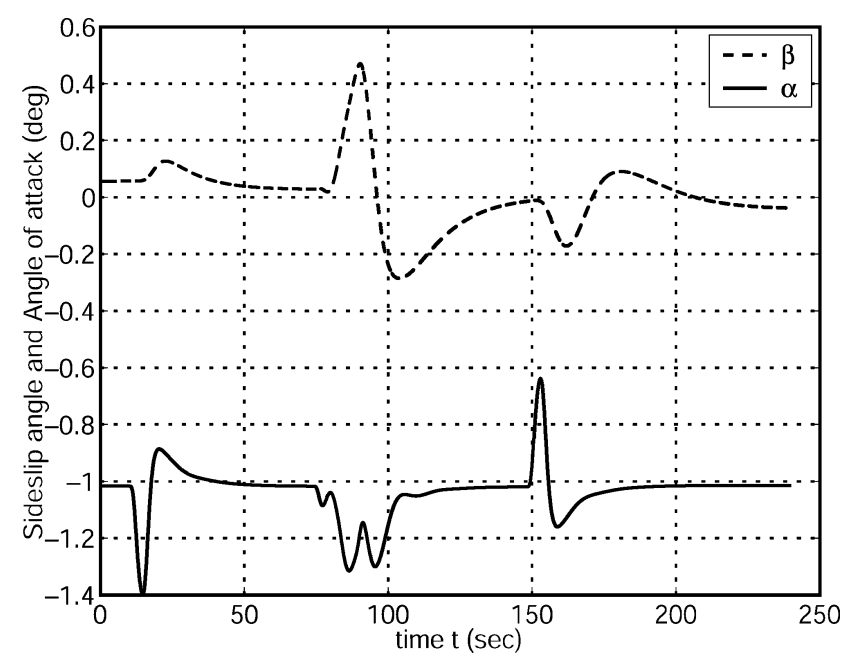

Fig. 15 Angle-of-attack and sideslip-angle variation.

ponents and wind gradients in effect) presented here. In all of the cases, perfect position tracking was achieved. The explanation for why $\phi$ has zero steady-state value while $\psi$ does not is that an integral control is used to ensure zero steady-state error in bank angle as shown in Sec. IV.D. Without this integral control, both $\psi$ and $\phi$ would go to nonzero values at the steady-state condition if wind gradients are present. In the case in which there is no wind gradient effect, both $\psi$ and $\phi$ would go to zero at steady state even without the integral control. Adding another integral control for $\psi$ to have a zero steady-state value would make the closed-loop system uncontrollable because, then, the number of outputs to regulate would become more than the number of inputs available.

An explanation for $\psi$ to have nonzero steady-state value solely caused by the wind gradients is that the wind components cause a disturbance in the translational motion (which is completely controlled to achieve precise position tracking), whereas the wind gradients cause a disturbance in the rotational motion (which cannot be completely regulated by this controller).

Though the results of the simulation of just one reconfiguration maneuver have been presented, multiple simulations with various initial and final positions and various speeds of reconfiguration were performed, and the results seemed very satisfactory.

\section{Conclusions}

In this paper, the dynamics involved in the reconfiguration of an unmanned aerial vehicle (UAV) flying in close formation have been modeled and simulated using a set of nonlinear, six-degreeof-freedom equations of motion involving wind effect, written in the frame of the virtual leader (VL). The vortex effect terms have been computed based on an averaging method. An algorithm that can generate a safe and feasible reference trajectory for reconfiguration, given the number of the individual UAV that is to be repositioned and its initial and final position numbers, has been developed.

A multiloop nonlinear position-tracking controller has been designed so that each of the individual UAVs track their reference trajectories specified in the VL frame. Timescale decomposition is performed in the control design procedure. A combination of nonlinear feedback linearization (for the inner loop) and linear-quadraticregulator design with integral control (for the outer loop) have been used to design the overall nonlinear reconfiguration controller. The controller is not provided with the information of the vortex-induced wind velocity and gradient.

Formation-flight simulations conducted with two UAVs demonstrate a highly satisfactory performance of the nonlinear controller in both following the VL maneuvering and achieving a smooth reconfiguration of the follower UAV, even when the vortex-induced effects on the follower's dynamics are included in the simulation. The reconfiguration trajectory generation is parameterized so that 
the speed and shape of reconfiguration maneuvers can be varied while following the VL. The UAV undergoing reconfiguration is brought to the steady state at the end of the reconfiguration maneuver. The time history of the relative separations between the UAVs and the VL showed that there was sufficiently big clearance between the two UAVs throughout the reconfiguration.

Several possible future extensions of this work have been identified. First, the simulation would be performed with more than two (most likely six) UAVs flying in close formation. Hence, a scenario with dynamically changing leaders would be considered to study the vortex-induced effects.

A second interesting problem would be to conduct an analytical stability and robustness analysis of the control law that has been developed in this paper. Also the various aspects involved in the actual hardware implementation of the preceding controller are to be studied in greater detail.

Third, the vortex effect implementation method and the nonlinear position-tracking controller that have been tested in the context of close formation flight in this paper can be extended to other applications such as the study of in-flight or aerial refueling.

\section{Appendix A: Scalar Equations of Translational Motion with Wind Effect}

\section{Translational Kinematics}

$$
\begin{aligned}
\dot{x}= & V \cos \beta \cos \alpha \cos \theta \cos \psi+V \sin \beta(-\cos \phi \sin \psi \\
& +\sin \phi \sin \theta \cos \psi)+V \cos \beta \sin \alpha(\sin \phi \sin \psi \\
& +\cos \phi \sin \theta \cos \psi)+\left[(\cos \theta \cos \psi) W_{x}\right. \\
& +(-\cos \phi \sin \psi+\sin \phi \sin \theta \cos \psi) W_{y}(\sin \phi \sin \psi \\
& \left.+\cos \phi \sin \theta \cos \psi) W_{z}\right]-\dot{x}_{\mathrm{VL}} \\
\dot{y}= & V \cos \beta \cos \alpha \cos \theta \sin \psi+V \sin \beta(\cos \phi \cos \psi \\
& +\sin \phi \sin \theta \sin \psi)+V \cos \beta \sin \alpha(-\sin \phi \cos \psi \\
& +\cos \phi \sin \theta \sin \psi)+\left[(\cos \theta \sin \psi) W_{x}\right. \\
& +(\cos \phi \cos \psi+\sin \phi \sin \theta \sin \psi) W_{y}(-\sin \phi \cos \psi \\
& \left.+\cos \phi \sin \theta \sin \psi) W_{z}\right]-\dot{y}_{\mathrm{VL}} \\
\dot{z}= & V[-\cos \beta \cos \alpha \sin \theta+\sin \beta \sin \phi \cos \theta \\
& +\cos \beta \sin \alpha \cos \phi \cos \theta]+\left[(-\sin \theta) W_{x}+(\sin \phi \cos \theta) W_{y}\right. \\
& \left.+(\cos \phi \cos \theta) W_{z}\right]-\dot{z}_{\mathrm{VL}}
\end{aligned}
$$

Translational Dynamics

$$
\begin{aligned}
\dot{V}= & g \cos \theta \sin \beta \sin \phi+g \cos \beta(\cos \phi \cos \theta \sin \alpha-\cos \alpha \sin \theta) \\
& +(1 / m)[-D+T \cos (\alpha+\delta) \cos \beta] \\
& +\left[e s(1,1) W_{x}+e s(1,2) W_{y}+e s(1,3) W_{z}\right] \\
& -\left[e i(1,1) \dot{W}_{x}+e i(1,2) \dot{W}_{y}+e i(1,3) \dot{W}_{z}\right] \\
\dot{\beta}= & -r \cos \alpha+p \sin \alpha+(g / V)(-\cos \phi \cos \theta \sin \alpha \sin \beta \\
& +\cos \beta \cos \theta \sin \phi+\cos \alpha \sin \beta \sin \theta) \\
& -(1 / m V)[S+T \cos (\alpha+\delta) \sin \beta] \\
& +\left[e s(2,1) W_{x}+e s(2,2) W_{y}+e s(2,3) W_{z}\right] \\
& -\left[e i(2,1) \dot{W}_{x}+e i(2,2) \dot{W}_{y}+e i(2,3) \dot{W}_{z}\right]
\end{aligned}
$$

$$
\begin{aligned}
\dot{\alpha}= & q-(p \cos \alpha+r \sin \alpha) \tan \beta+(g \sec \beta / V)(\cos \alpha \cos \phi \cos \theta \\
& +\sin \alpha \sin \theta)-(\sec \beta / m V)[L+T \sin (\alpha+\delta)] \\
& +\left[e s(3,1) W_{x}+e s(3,2) W_{y}+e s(3,3) W_{z}\right] \\
& -\left[e i(3,1) \dot{W}_{x}+e i(3,2) \dot{W}_{y}+e i(3,3) \dot{W}_{z}\right]
\end{aligned}
$$

where

$$
\begin{aligned}
e s & =\mathcal{E}^{-1} \boldsymbol{S}\left(\omega_{B}\right) \\
e i & =\mathcal{E}^{-1} \\
& =\left[\begin{array}{ccc}
\cos \alpha \cos \beta & \sin \beta & \cos \beta \sin \alpha \\
-\frac{\cos \alpha \sin \beta}{V} & \frac{\cos \beta}{V} & -\frac{\sin \alpha \sin \beta}{V} \\
-\frac{\sec \beta \sin \alpha}{V} & 0 & \frac{\cos \alpha \sec \beta}{V}
\end{array}\right]
\end{aligned}
$$

\section{Appendix B: Numerical Data}

Note: The following data apply identically to each aircraft considered in the simulation. Whereas all of the angles are provided in degrees, in the simulation the values in radians are used.

\section{Simulation Parameters}

Formation-flight velocity $V: 150 \mathrm{~m} / \mathrm{s}$

Formation-flight altitude (for air): $3000 \mathrm{~m}$ above sea level

\section{Aircraft Parameters}

Mass $m: 2.5493 \times 10^{5} \mathrm{~N}$

Wing area $\mathcal{S}: 511 \mathrm{~m}^{2}$

Wing mean chord $\bar{c}: 8.32 \mathrm{~m}$

Wing span $b: 59.74 \mathrm{~m}$

Moment arm of thrust around body $y$ axis $\Delta z: 3.7184 \mathrm{~m}$

Thrust deflection angle $\delta: 1 \mathrm{deg}$

Diameter of the fuselage $D_{F}: 10 \mathrm{~m}$

Length of the fuselage $L_{F}: 45 \mathrm{~m}$

$x$ distance between the c.g. of the aircraft and the aerodynamic center of the wing: $0 \mathrm{~m}$

$z$ distance between the c.g. of the aircraft and the aerodynamic center of the wing: $0 \mathrm{~m}$

Dihedral angle $\zeta: 0$ deg

Sweepback angle at quarter-chord $\lambda: 0 \mathrm{deg}$

Moments of inertia, $\mathrm{Nm}^{2}: I_{x x}=1.86 \mathrm{e} 7 ; I_{y y}=4.14 \mathrm{e} 7 ; I_{z z}=$ $5.83 \mathrm{e} 7 ; I_{x z}=1.13 \mathrm{e} 6$

Aerodynamic coefficients: $C_{L_{0}}=.92 ; C_{L_{\alpha}}=5.67 ; C_{L_{\alpha^{2}}}=-5.95$; $C_{L_{q}}=5.65 ; C_{L_{\delta_{e}}}=0.36 ; \alpha_{\text {ref }}=13 \mathrm{deg} ; C_{D_{0}}=0.0751 ; C_{D_{\alpha^{2}}}=$ $3.7642 ; C_{S_{0}}=0 ; C_{S_{\beta}}=-1.08 ; C_{S_{\delta_{r}}}=0.179 ; C_{\mathcal{L}_{0}}=0 ; C_{\mathcal{L}_{\delta_{a}}}=$ $.053 ; C_{\mathcal{L}_{\delta_{r}}}=0 ; C_{\mathcal{L}_{\beta}}=-0.281 ; C_{\mathcal{L}_{p}}=-0.502 ; C_{\mathcal{L}_{r}}=0.195 ;$ $C_{\mathcal{M}_{0}}=0 ; \quad C_{\mathcal{M}_{\alpha}}=-1.45 ; \quad C_{\mathcal{M}_{\delta_{e}}}=-1.4 ; \quad C_{\mathcal{M}_{q}}=-21.4 ;$ $C_{\mathcal{N}_{0}}=0 ; C_{\mathcal{N}_{\delta_{a}}}=.0083 ; C_{\mathcal{N}_{\delta_{r}}}=-0.113 ; C_{\mathcal{N}_{\beta}}=0.184 ; C_{\mathcal{N}_{p}}=$ $-0.222 ; C_{\mathcal{N}_{r}}=-0.36$

\section{Engine Parameters}

Time constant of the engine $\tau: 3$

Maximum thrust $T_{\max }: 9.3 \mathrm{e} 5 \mathrm{~N}$

\section{Control Parameters}

Nominal Conditions

$V_{0}=150 \mathrm{~m} / \mathrm{s} ; \beta_{0}=0 \mathrm{deg} ; \alpha_{0}=-1.01811701818346 \mathrm{deg} ; p_{0}=$ $0 \mathrm{deg} / \mathrm{s} ; \quad q_{0}=0 \mathrm{deg} / \mathrm{s} ; \quad r_{0}=0 \mathrm{deg} / \mathrm{s} ; \quad \psi_{0}=0 \mathrm{deg} ; \quad \theta_{0}=$ $-1.01811701818346 \mathrm{deg} ; \quad \phi_{0}=0 \mathrm{deg} ; \quad \delta_{a_{0}}=0 \mathrm{deg} ; \quad \delta_{e_{0}}=$ $2.44984018390870 \mathrm{deg} ; \delta_{r_{0}}=0 \mathrm{deg} ; \xi_{t_{0}}=0.42864572758644$

\section{LQR Design Matrices}

$\boldsymbol{Q}=\operatorname{diag}\left(\left[\begin{array}{llllllllllllll}1 & 100 & 100 & 1 & 1 & 1 & 100 & 100 & 0.01 & 1 & 1 & 1 & 1 & 1\end{array}\right]\right)$

$\boldsymbol{R}=\operatorname{diag}\left(\left[\begin{array}{llll}.01 & 100 & 100 & 500\end{array}\right]\right)$ 


\section{Trajectory Generation Parameters}

(same filter coefficients applied to $x, y$, and $z$ position commands) $k_{10}=0.1 ; k_{11}=1.3 ; k_{12}=3.3 ; k_{13}=3.1$

\section{References}

${ }^{1}$ Blake, W., and Multhopp, D., "Design, Performance and Modeling Considerations for Close Formation Flight," Proceedings of the 1998 AIAA Guidance, Navigation, and Control Conference, AIAA, Reston, VA, 1998, pp. 476-486.

${ }^{2}$ Dargan, J., Pachter, M., and D’Azzo, J., "Automatic Formation Flight Control," Proceedings of the 1992 AIAA Guidance, Navigation, and Control Conference, AIAA, Washington, DC, 1992, pp. 838-857.

${ }^{3}$ Fierro, R., Belta, C., Desai, J., and Kumar, V., "On Controlling Aircraft Formations," Proceedings of the 40th IEEE Conference on Decision and Control, Vol. 2, Inst. of Electrical and Electronics Engineers, Arlington, VA, 2001, pp. 1065-1070.

${ }^{4}$ Koo, T., and Shahruz, S., "Formation of a Group of Unmanned Aerial Vehicles (UAVs)," Proceedings of the American Control Conference, Inst. of Electrical and Electronics Engineers, Arlington, VA, 2001, pp. 69-74.

${ }^{5}$ Giuletti, F., Pollini, L., and Innocenti, M., "Autonomous Formation Flight," IEEE Control Systems Magazine, Vol. 20, No. 6, Dec. 2000, pp. 34-44.

${ }^{6}$ Pollini, L., Giuletti, F., and Innocenti, M., "Robustness to Communication Failures Within Formation Flight," Proceedings of the American Con- trol Conference, Inst. of Electrical and Electronics Engineers, Arlington, VA, 2002, pp. 2860-2866.

${ }^{7}$ Chichka, D. F., Speyer, J., and Park, C., "Peak-Seeking Control with Application to Formation Flight," Proceedings of the 38th IEEE Conference on Decision and Control, Vol. 3, Inst. of Electrical and Electronics Engineers, Arlington, VA, 1999, pp. 2463-2470.

${ }^{8}$ Pan, H., and Kapila, V., "Adaptive Nonlinear Control for Spacecraft Formation Flying with Coupled Translational and Attitude Dynamics," Proceedings of the 40th IEEE Conference on Decision and Control, Vol. 3, Inst. of Electrical and Electronics Engineers, Arlington, VA, 2001, pp. 2057-2062.

${ }^{9}$ Dogan, A., "Guidance Strategies for Microburst Escape," Ph.D. Dissertation, Dept. of Aerospace Engineering, Univ. of Michigan, Ann Arbor, MI, May 2000.

${ }^{10}$ Dogan, A., and Kabamba, P., "Escaping a Microburst with Turbulence: Altitude, Dive and Pitch Guidance Strategies," Journal of Aircraft, Vol. 37, No. 3, 2000, pp. 417-426.

${ }^{11}$ Pamadi, B. N., Performance, Stability, Dynamics, and Control of Airplanes, AIAA, Reston, VA, 2004, Chap. 4.

${ }^{12}$ Nelson, R. C., Flight Stability and Automatic Control, McGraw-Hill, New York, 1998, Chap. 1.

${ }^{13}$ Venkataramanan, S., Dogan, A., and Blake, W., "Vortex Effect Modelling in Aircraft Formation Flight," Proceedings of the AIAA AFM Conference, AIAA, Reston, VA, 2003.

${ }^{14}$ Venkataramanan, S., "Dynamics and Control of Multiple UAVs Flying in Close Proximity," M.S. Thesis, Dept. of Mechanical and Aerospace Engineering, Univ. of Texas, Arlington, TX, Aug. 2004. 